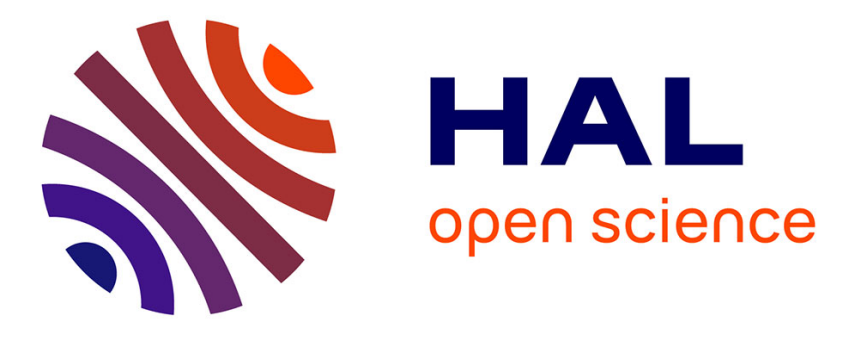

\title{
Study of a 1,6-hydride shift in an open chain of hydroxylactam-triarylcarbinols
}

Pascal Pigeon, Aïcha Mamouni, Jana Sikoraiova, Stefan Marchalin, Bernard Decroix

\section{- To cite this version:}

Pascal Pigeon, Aïcha Mamouni, Jana Sikoraiova, Stefan Marchalin, Bernard Decroix. Study of a 1,6-hydride shift in an open chain of hydroxylactam-triarylcarbinols. Tetrahedron, 2001, 57 (23), pp.4939-4943. 10.1016/S0040-4020(01)00437-9 . hal-01230408

\section{HAL Id: hal-01230408 https://hal.science/hal-01230408}

Submitted on 6 Feb 2018

HAL is a multi-disciplinary open access archive for the deposit and dissemination of scientific research documents, whether they are published or not. The documents may come from teaching and research institutions in France or abroad, or from public or private research centers.
L'archive ouverte pluridisciplinaire HAL, est destinée au dépôt et à la diffusion de documents scientifiques de niveau recherche, publiés ou non, émanant des établissements d'enseignement et de recherche français ou étrangers, des laboratoires publics ou privés. 


\title{
Study of a 1,6-hydride shift in an open chain of hydroxylactam-triarylcarbinols
}

Pascal Pigeon, ${ }^{\mathrm{a}}$ Aïcha Mamouni, ${ }^{\mathrm{a}}$ Jana Sikoraiova, ${ }^{\mathrm{b}}$ Stefan Marchalin ${ }^{\mathrm{b}}$ and Bernard Decroix ${ }^{\mathrm{a}}{ }^{*}$

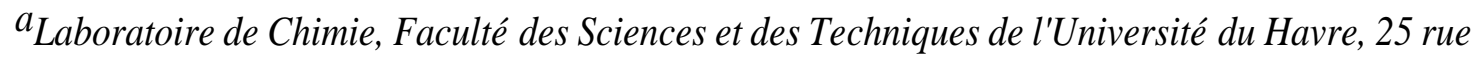

Philippe Lebon, BP 540, 76058 Le Havre Cedex, France

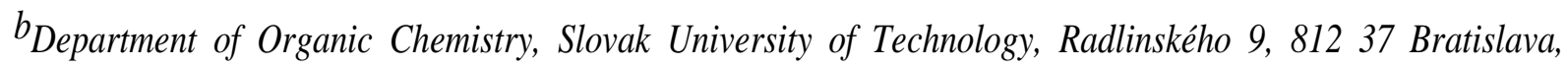
Slovak Republic

Keywords: iminium salts; isoindole; oxazepines; polycyclic heterocyclic compounds.

* Corresponding author. Tel.: +33-02-32-74-43-95; fax: +33-02-32-74-43-91; e-mail: bernard.decroix@univ-lehavre.fr

\begin{abstract}
Hydroxylactam-alcohols 5a,b,d (isoindole series), under acidic treatment gave phthalimides 7a,b,d via a hydride shift induced by an intramolecular $\pi$-stacking (benzene-benzene or benzene-thiophene interaction) which did not occur in $\mathbf{1 0 a}$ (succinimide series).
\end{abstract}

The pinacol rearrangement is a well-known reaction and it has been demonstrated that the hydrogen has a greater migratory aptitude than a phenyl group. Although direct nucleophilic rearrangement over distances greater than 1,2 are rare, a shift across a ring of 8-11 members ${ }^{1,2}$ is still possible as exemplified with cyclodecanediol $\mathbf{1}^{3}$ (Scheme 1$)$.

Based on these observations we intended to study structures of type $\mathbf{I}$, because during our investigations on the synthesis of polyheterocycles containing an isoindole moiety, we have prepared hydroxylactam-alcohols 5a-d. Actually, in an acidic medium the hydroxylactam is a precursor of an $\mathrm{N}$-acyliminium ion, ${ }^{4}$ and the triarylcarbinol can also give a carbocation. Consequently, it was reasonable to investigate the possibilities of a hydrogen migration from the hydroxylactam to the carbinol.

In a diol, as might be expected, the leaving hydroxy group is the one which leads to the more 
stable carbocation. We have recently described that an $\mathrm{N}$-acyliminium ion reacts easily with an alcohol or a thiol. ${ }^{5}$ Thus from compound $\mathbf{5 a}$, we expected either the formation of an ether or a nucleophilic pinacol rearrangement if the geometry of the structure permits the transfer of an atom similar to the one observed in cyclodecanediol 1. The starting hydroxylactamalcohols 5a-c were obtained in good yields by reducing the phthalimide-esters $\mathbf{3 a}-\mathbf{b}^{6,7}$ with sodium borohydride and by subsequent addition of an excess of phenylmagnesium bromide (Scheme 2). Thus, when 5a was subjected to a catalytic amount of $p$-toluenesulfonic acid (PTSA) in refluxing toluene for $2 \mathrm{~h}$, a mixture of ether $6 \mathbf{a}(80 \%)$ and imide 7a (20\%) was formed. Compounds 5a, 6a and 7a were easily differentiated by the analysis of their NMR $\left({ }^{1} \mathrm{H}\right.$ and $\left.{ }^{13} \mathrm{C}\right)$ spectra. Due to the presence of a stereogenic center, methylene protons $\left(\mathrm{N}-\mathrm{CH}_{2}\right)$ and carbon atoms of the two phenyl groups are not equivalent for $5 \mathbf{a}$ and $\mathbf{6 a}$ in contrast to $7 \mathbf{a}$. When a catalytic amount of triftuoroacetic acid (TFA) in place of PTSA was used during 30 min, we observed the ether $6 \mathbf{a}$ accompanied with traces of 7a. The imide 7a was obtained from $5 \mathbf{a}$ or $6 \mathbf{a}$ as the sole product when the reaction was carried out for 2 days with an excess of TFA. This behavior was similar to a pinacol rearrangement. To prove that the product $7 \mathbf{a}$ resulted of a hydrogen migration we prepared the deuterated hydroxylactam $\mathbf{5 a}$. The latter resulted of the reduction of $\mathbf{4 a}$ in the presence of deuterated sodium borohydride. The deuterated compound 5a gave the deuterated phthalimide 7a. A NMR analysis revealed the absence of hydrogen in the $\alpha$-position to the two phenyl groups compared to that observed in the non-deuterated product $7 \mathbf{a}(\delta=6.22 \mathrm{ppm})$. The hydrogen migration can be rationalized as depicted in Scheme 3. The more stable carbocation A was first formed, followed by a transfer of deuterium which led to a new carbocation $\mathbf{B}$ stabilized by both a nitrogen and an oxygen atom. Finally, loss of a proton provided phthalimide $\mathbf{7 a}$. This hydride transfer was supposed to be due to the geometry of the carbocation $\mathbf{A}$.

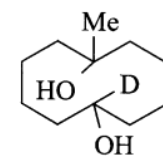

1

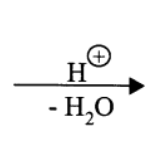

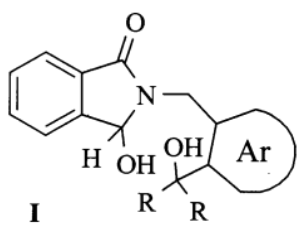

Scheme 1. 

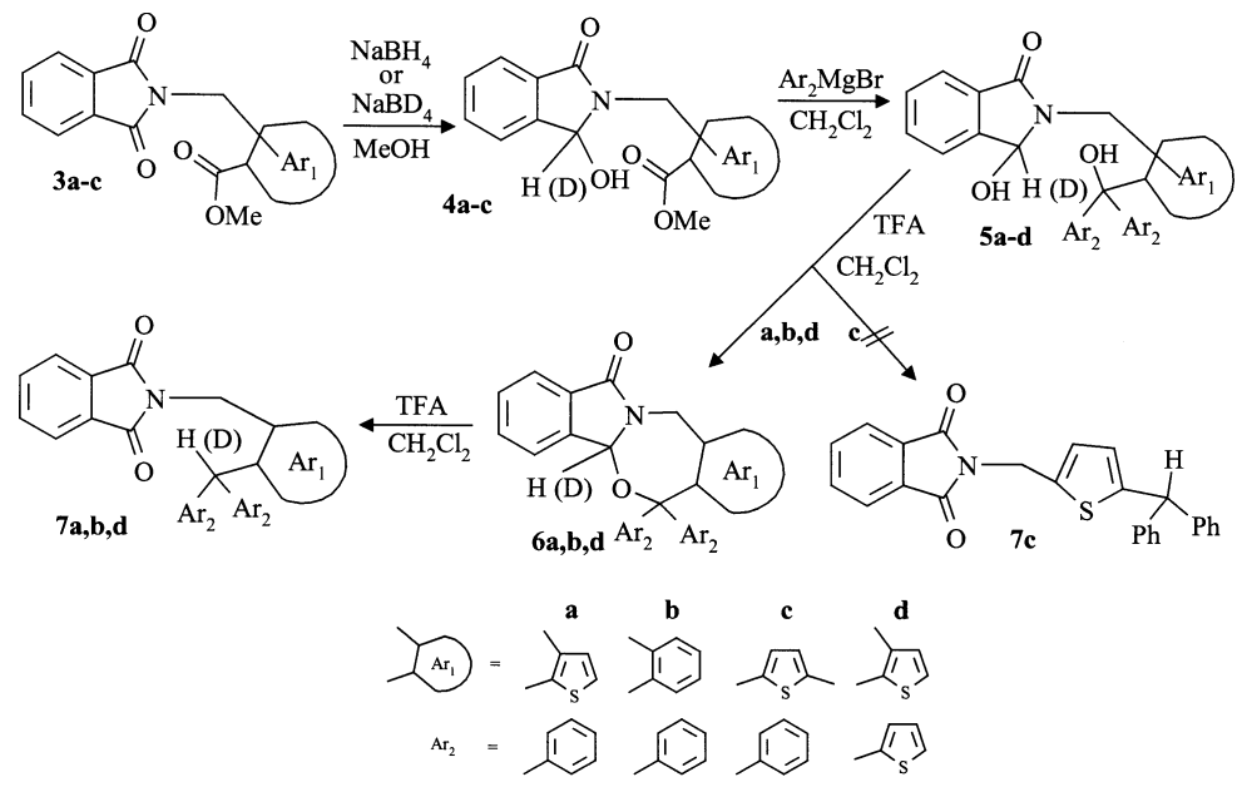

Scheme 2.

Actually, analysis of molecular models showed a proximity between the hydrogen (or deuterium) atom and the positive carbon of carbocation $\mathbf{A}$ when one of the two phenyl groups was parallel to the benzene ring of the isoindole moiety. To confirm this result, we tested the diol $\mathbf{5 b}$ (the thiophene ring of $\mathbf{5 a}$ has been replaced by a benzene ring). Under the same acidic conditions (TFA, dichloromethane) $\mathbf{5 b}$ gave the cyclic ether $\mathbf{6 b}$ and the imide $\mathbf{7 b}$. To show that the $\pi$-stacking ${ }^{8-10}$ seems to be necessary we prepared the alcohol $\mathbf{5 c}$. Treated in the same manner as above (TFA, $\mathrm{CH}_{2} \mathrm{Cl}_{2}$ or PTSA, toluene, reflux) $\mathbf{5 c}$ gave no reaction, the starting alcohol being recovered. In that case the geometry of the molecule did not permit either a cyclodehydration or a hydrogen migration leading to $\mathbf{7 c}$.

Another argument in favor of a $\pi$-stacking was obtained during the investigation of diol 10a (Scheme 4). We tested the tetramethylsuccinimide in place of the isoindole moiety (the methyl groups in $\mathbf{1 0}$ prevented loss of a proton in the $\alpha$ position of the hydroxy group leading to an enamide). The imide $\mathbf{8}$ was prepared via the alkylation of the tetramethylsuccinimide with the methyl 3-bromomethylthiophene-2-carboxylate in toluene using potassium carbonate as the base. In contrast to the phthalimide series $^{6}$ phenylmagnesium bromide in dichloromethane selectively reacted with the ester function to give the alcohol-imide 9. Reduction of 9 with sodium borohydride at $0^{\circ} \mathrm{C}$ in methanol as for $\mathbf{4 a - d}$ gave the diol 10a $(85 \%)$ accompanied with the ether $\mathbf{1 0 b}(15 \%)$ in a $95 \%$ yield. The mixture $\mathbf{1 0 a} / \mathbf{1 0 b}$ was not separated and was used in the next step without further purification. In a similar manner as above under acidic treatment 10a (and 10b) gave the ether $\mathbf{1 1}$ (PTSA, dichloromethane) as the 
single product because the imide 12 (TFA, dichloromethane) was not observed. In the latter case there is no $\pi-\pi$ interaction and furthermore the imide is shielded by the four methyl groups.

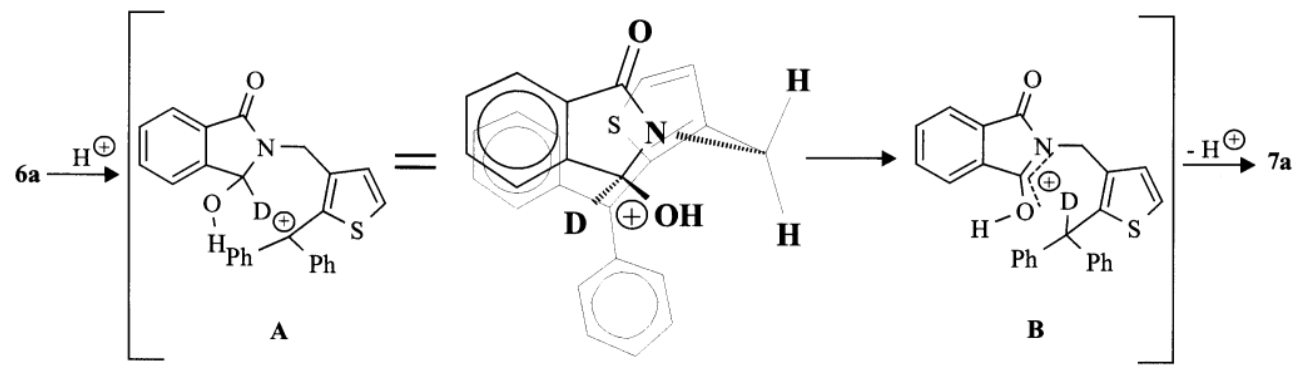

Scheme 3.

As another example we prepared the oxazepine $\mathbf{6 d}$ and the imide $\mathbf{7 d}$ from diol $\mathbf{5 d}$. This diol resulted from the action of 6 equiv. of thien-2-ylmagnesium bromide onto the ester 4a. The replacement of the two benzene rings $(\mathbf{5 a}, \mathbf{b})$ by two thiophene rings $(\mathbf{5} \mathbf{d})$ has modified the $\pi-\pi$ interaction. Actually the hydride transfer was carried out in two days for 5a,b while after seven days only $35 \%$ of $\mathbf{5 d}$ had reacted with the formation of $13 \%$ of $\mathbf{7 d}$ accompanied with degradation products. Furthermore, as in benzene series we did not observe a reaction between the $\mathrm{N}$-acyliminium ion and one of the two thiophene rings whatever the conditions of the reaction (temperature, time).

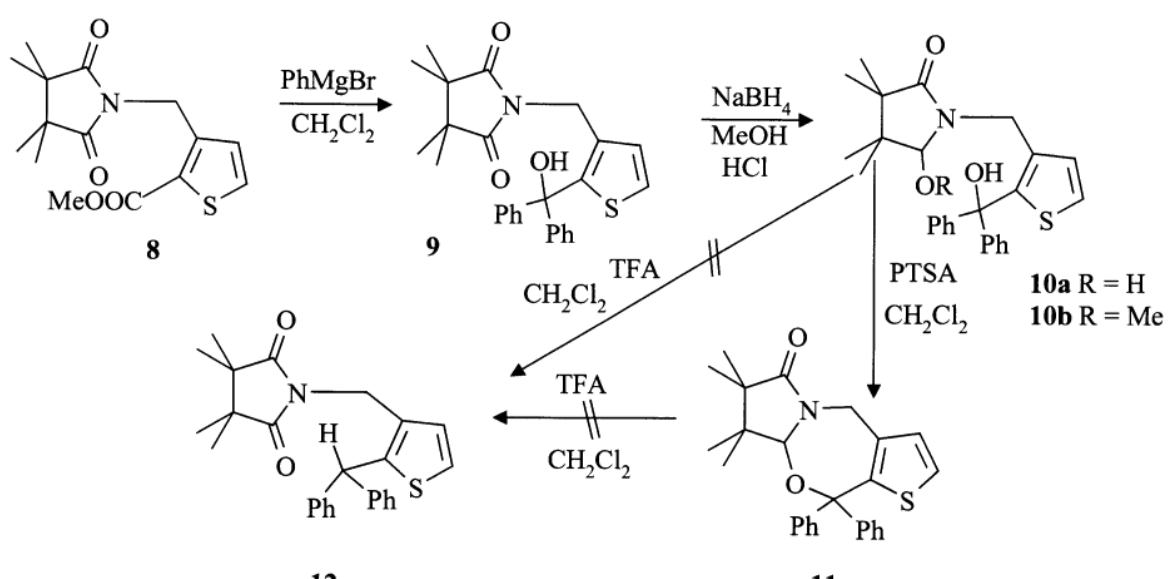

Scheme 4.

In conclusion we have described an interesting hydride transfer between a hydroxylactam and an alcohol in a non-cyclic product. A $\pi$-stacking in the intermediate carbocation seems to be necessary for this transfer. Further studies are in progress to investigate scope and limitations of this new type of hydrogen migration. 


\section{Experimental}

\subsection{General}

Melting points are uncorrected. The infrared spectra of solids (potassium bromide) were recorded on a Perkin- Elmer FTIR paragon 1000 spectrometer. The ${ }^{1} \mathrm{H}$ and ${ }^{13} \mathrm{C}$ NMR spectra were recorded on a Bruker AC-200 (200 MHz) instrument in deuterochloroform solution and chemical shifts $(\delta)$ are expressed in ppm relative to internal TMS. Thin layer chromatography was performed on precoated plates of silica gel 60 F254 (Merck) and the spots visualized using an ultraviolet lamp or iodine vapor. E. Merck silica gel 60 F (70-300 mesh) was used for column chromatography. The elemental analyses were carried out by the microanalysis laboratory of INSA at Rouen, F 76130 Mt. St. Aignan, France. Compounds 4a,b were prepared as indicated in Refs. 6 and 7.

\subsubsection{2,3-Dihydro-3-hydroxy-2-[2-(diphenylhydroxymethyl)thien-3-ylmethyl]-1H-}

isoindol-1-one (5a). To a freshly prepared solution of phenylmagnesium bromide $(60 \mathrm{~mL}$ of $1 \mathrm{M}$ solution in ether) in dry dichloromethane $(60 \mathrm{~mL})$ was added portionwise solid hydroxylactam 4a $(3.03 \mathrm{~g}, 10 \mathrm{mmol})$ at room temperature with stirring. Stirring was continued for $3 \mathrm{~h}$. The solution was then carefully poured into a $10 \%$ solution of ammonium chloride. The mixture was decanted and the aqueous layer was extracted with dichloromethane. The combined organic layers were dried on magnesium sulfate and filtered. The solution was concentrated under reduced pressure. The residue was chromatographed on silica gel (dichloromethane until the biphenyl was removed, then dichloromethane/acetone 90/10). The diol was recrystallized from ethanol to furnish pure product 5a. Yield 56\%; mp $162^{\circ} \mathrm{C}$; IR: $3368(\mathrm{OH}), 3330(\mathrm{OH}), 1660(\mathrm{C}=\mathrm{O}) \mathrm{cm}^{-1}$; ${ }^{1} \mathrm{H}$ NMR: $\delta 4.14(\mathrm{~d}, J=15 \mathrm{~Hz}, 1 \mathrm{H}$, $\left.\mathrm{CH}_{2}\right), 4.22(\mathrm{~s}, 1 \mathrm{H}, \mathrm{OH}), 4.49$ (d, J=15 Hz, 1H, $\left.\mathrm{CH}_{2}\right), 5.59$ (s, 1H, OH), 5.67 (s, 1H, CH), 6.98

(d, J=5 Hz, 1H, $\left.\mathrm{H}_{\text {thiophene }}\right), 7.03\left(\mathrm{~d}, J=5 \mathrm{~Hz}, 1 \mathrm{H}, \mathrm{H}_{\text {thiophene }}\right), 7.06-7.63\left(\mathrm{~m}, 14 \mathrm{H}, \mathrm{H}_{\text {arom }}\right) ;{ }^{13} \mathrm{C}$ NMR: $\delta 37.2\left(\mathrm{CH}_{2}\right), 79.0(\mathrm{C}), 81.5(\mathrm{CH}), 123.2(\mathrm{CH}), 123.3(\mathrm{CH}), 124.5(\mathrm{CH}), 127.2(2 \mathrm{CH})$, $127.5(2 \mathrm{CH}), 127.6(\mathrm{CH}), 127.7(\mathrm{CH}), 127.9(4 \mathrm{CH}), 129.7(\mathrm{CH}), 130.5(\mathrm{CH}), 131.2(\mathrm{C})$, 132.3 (CH), 134.3 (C), 143.1 (C), 146.7 (C), 147.0 (C), 148.5 (C), 167.3 (CO). Anal. Calcd for $\mathrm{C}_{26} \mathrm{H}_{21} \mathrm{NO}_{3} \mathrm{~S}$ : C, 73.05; H, 4.95; N, 3.28. Found: C, 72.78; H, 4.89; N, 3.34.

\subsubsection{2,3-Dihydro-3-hydroxy-2-[2-(diphenylhydroxymethyl)phenylmethyl]-1H-}


isoindol-1-one (5b). This compound was prepared from $\mathbf{4 b}$ using the same procedure as for 5a. Yield 59\%; mp $118^{\circ} \mathrm{C}$; IR: $3306(\mathrm{OH}), 1680(\mathrm{C}=\mathrm{O}) \mathrm{cm}^{-1} ;{ }^{1} \mathrm{H}$ NMR: $\delta 4.40(\mathrm{~d}, J=15 \mathrm{~Hz}$, $\left.1 \mathrm{H}, \mathrm{CH}_{2}\right), 4.63\left(\mathrm{~d}, J=15 \mathrm{~Hz}, 1 \mathrm{H}, \mathrm{CH}_{2}\right), 4.73$ (d, J=7 Hz, 1H, OH), 5.59 (d, J=7 Hz, 1H, CH), $6.56\left(\mathrm{~d}, J=8 \mathrm{~Hz}, 1 \mathrm{H}, \mathrm{H}_{\text {arom }}\right), 6.92-7.52\left(\mathrm{~m}, 16 \mathrm{H}, \mathrm{H}_{\text {arom }}\right), 7.61\left(\mathrm{~d}, J=7 \mathrm{~Hz}, 1 \mathrm{H}, \mathrm{H}_{\text {arom }}\right) ;{ }^{13} \mathrm{C}$ NMR: $\delta 41.2\left(\mathrm{CH}_{2}\right), 81.2(\mathrm{CH}), 82.9(\mathrm{C}), 123.0(2 \mathrm{CH}), 126.3(\mathrm{CH}), 127.0(\mathrm{CH}), 127.1(\mathrm{CH})$, $127.3(2 \mathrm{CH}), 127.7(2 \mathrm{CH}), 127.8(2 \mathrm{CH}), 127.9(2 \mathrm{CH}), 128.2(\mathrm{CH}), 129.3(\mathrm{CH}), 129.8$ $(\mathrm{CH}), 131.3(\mathrm{CH}), 131.4(\mathrm{C}), 131.9(\mathrm{CH}), 137.1(\mathrm{C}), 143.0(\mathrm{C}), 144.2$ (C), 146.9 (C), 147.0 (C), 167.5 (CO). Anal. Calcd for $\mathrm{C}_{28} \mathrm{H}_{23} \mathrm{NO}_{3}$ : C, 79.79; H, 5.50; N, 3.32. Found: C, 79.34; H, $5.62 ; \mathrm{N}, 3.15$.

\subsubsection{2,3-Dihydro-3-hydroxy-2-[2-(di(thien-2-yl)hydroxymethyl)thien-3-ylmethyl]-1H-}

isoindol-1-one (5d). This compound was prepared from $\mathbf{4 a}$ using the same procedure as for 5a,b but with 2-thienylmagnesium bromide. Yield 64\%; mp $202^{\circ} \mathrm{C}$; IR: $3422(\mathrm{OH}), 1656$ $(\mathrm{C}=\mathrm{O}) \mathrm{cm}^{-1}$; ${ }^{1} \mathrm{H}$ NMR: $\delta 3.50$ (s broad, $\left.1 \mathrm{H}, \mathrm{N}-\mathrm{C}-\mathrm{OH}\right), 4.37$ (d, J=15 Hz, 1H, $\left.\mathrm{CH}_{2}\right), 4.64$ (d, $J=15 \mathrm{~Hz}, 1 \mathrm{H}, \mathrm{CH}_{2}$ ), 5.70 (s broad, 1H, N-CH), 6.46 (s broad, 1H, OH), 6.80-6.98 (m, 4H, $\left.\mathrm{H}_{\text {thiophene }}\right), 7.01$ (d, J=5 Hz, 1H, $\left.\mathrm{H}_{3}\right), 7.08\left(\mathrm{~d}, J=5 \mathrm{~Hz}, 1 \mathrm{H}, \mathrm{H}_{2}\right), 7.18-7.58$ (m, 5H, $\left.3_{\text {Harom }}+2 \mathrm{H}_{\text {thiophene }}\right), 7.64\left(\mathrm{~d}, J=7 \mathrm{~Hz}, 1 \mathrm{H}, \mathrm{H}_{\text {arom }}\right) ;{ }^{13} \mathrm{C} \mathrm{NMR:} \delta 37.5\left(\mathrm{CH}_{2}\right), 74.4(\mathrm{C}), 81.2(\mathrm{CH})$, $122.4(\mathrm{CH}), 123.6(\mathrm{CH}), 124.0(\mathrm{CH}), 125.6(\mathrm{CH}), 125.7(2 \mathrm{CH}), 125.8(\mathrm{CH}), 126.5(2 \mathrm{CH})$, $129.1(\mathrm{CH}), 129.4(\mathrm{CH}), 131.1(\mathrm{C}), 132.1(\mathrm{CH}), 135.4(\mathrm{C}), 145.0(\mathrm{C}), 145.8(\mathrm{C}), 152.1(\mathrm{C})$, 152.2 (C), 166.6 (CO). Anal. Calcd for $\mathrm{C}_{22} \mathrm{H}_{17} \mathrm{NO}_{3} \mathrm{~S}_{3}$ : C, 60.11; H, 3.90; N, 3.19. Found: C, $60.02 ; \mathrm{H}, 3.85 ; \mathrm{N}, 3.24$.

\subsubsection{4,12-Dihydro-12,12-diphenylthieno[ $\left[3^{\prime}, 2^{\prime}: 5,6\right][1,3]$ oxazepino $[2,3-a]$ isoindol-6}

$(\mathbf{1 0 b H})$-one (6a). A solution of compound $5 \mathbf{a}(10 \mathrm{mmol})$, a catalytic amount of PTSA in dichloromethane $(20 \mathrm{~mL})$ was stirred for $30 \mathrm{~min}$ (reaction was monitored by TLC). The solution was washed successively with a saturated sodium hydrogen carbonate solution, then with water and was dried (magnesium sulfate) and filtered. The solution was concentrated under reduced pressure and the residue was recrystallized from ethanol to furnish pure 6a: Yield 88\%; mp $178^{\circ} \mathrm{C}$; IR: $1707(\mathrm{C}=\mathrm{O}) \mathrm{cm}^{-1}$; ${ }^{1} \mathrm{H}$ NMR: $\delta 4.19\left(\mathrm{~d}, J=16 \mathrm{~Hz}, 1 \mathrm{H}, \mathrm{H}_{4}\right), 5.36(\mathrm{~d}$, $\left.J=16 \mathrm{~Hz}, 1 \mathrm{H}, \mathrm{H}_{4}\right), 6.01\left(\mathrm{~s}, 1 \mathrm{H}, \mathrm{H}_{10 \mathrm{~b}}\right), 7.05\left(\mathrm{~d}, 1=5 \mathrm{~Hz}, 1 \mathrm{H}, \mathrm{H}_{3}\right), 7.11\left(\mathrm{~d}, J=5 \mathrm{~Hz}, 1 \mathrm{H}, \mathrm{H}_{2}\right)$, 7.20-7.57 (m, 13H, $\left.\mathrm{H}_{\text {arom }}\right), 7.78$ (d, J=7 Hz, $\left.1 \mathrm{H}, \mathrm{H}_{7}\right) ;{ }^{13} \mathrm{C} \mathrm{NMR}: \delta 40.8\left(\mathrm{CH}_{2}\right), 84.7(\mathrm{CH}), 86.3$ (C), $123.2(\mathrm{CH}), 123.5(\mathrm{CH}), 124.7(\mathrm{CH}), 127.5(2 \mathrm{CH}), 127.8(2 \mathrm{CH}), 128.0(\mathrm{CH}), 128.7$ $(\mathrm{CH}), 128.8(2 \mathrm{CH}), 129.1(\mathrm{CH}), 129.4(2 \mathrm{CH}), 129.6(\mathrm{CH}), 131.7(\mathrm{C}), 132.0(\mathrm{CH}), 135.3(\mathrm{C})$, 141.4 (C), 142.6 (C), 144.7 (C), 146.6 (C), 166.5 (CO). Anal. Calcd for $\mathrm{C}_{26} \mathrm{H}_{19} \mathrm{NO}_{2} \mathrm{~S}$ : C, 
76.26; H, 4.68; N, 3.42. Found: C, 75.89; H, 4.81; N, 3.28.

2.1.5. 5,13-Dihydro-5,5-diphenylisoindolo[1,2-c][2,4]benzoxazepin-11(6aH)-one (6b). This compound was prepared from $\mathbf{5 b}$ using the same procedure as for 5a. Yield 93\%; mp 175 ${ }^{\circ} \mathrm{C}$; IR: $1677(\mathrm{C}=\mathrm{O}) \mathrm{cm}^{-1} ;{ }^{1} \mathrm{H}$ NMR: $\delta 4.48\left(\mathrm{~d}, J=15 \mathrm{~Hz}, 1 \mathrm{H}, \mathrm{H}_{5}\right), 4.65(\mathrm{~d}, J=15 \mathrm{~Hz}, 1 \mathrm{H}$, $\left.\mathrm{H}_{5}\right), 6.21$ (s, 1H, $\left.\mathrm{H}_{11 \mathrm{~b}}\right), 6.61$ (d, J=8 Hz, 1H, $\left.\mathrm{H}_{\text {arom }}\right), 7.05-7.61\left(\mathrm{~m}, 16 \mathrm{H}, \mathrm{H}_{\text {arom }}\right), 7.78$ (d, J=6 $\left.\mathrm{Hz}, 1 \mathrm{H}, \mathrm{H}_{8}\right) ;{ }^{13} \mathrm{C}$ NMR: $\delta 45.8\left(\mathrm{CH}_{2}\right), 83.3(\mathrm{CH}), 89.1(\mathrm{C}), 123.0(\mathrm{CH}), 123.0(\mathrm{CH}), 127.2$ $(\mathrm{CH}), 127.4(3 \mathrm{CH}), 127.7(2 \mathrm{CH}), 127.9(\mathrm{CH}), 128.2(2 \mathrm{CH}), 128.5(\mathrm{CH}), 129.1(2 \mathrm{CH})$, $129.3(\mathrm{CH}), 130.2(\mathrm{CH}), 131.4(\mathrm{CH}), 131.6(\mathrm{CH}), 132.2(\mathrm{C}), 135.3(\mathrm{C}), 142.1(\mathrm{C}), 142.2(\mathrm{C})$, 142.4 (C), 145.7 (C), 165.8 (CO). Anal. Calcd for $\mathrm{C}_{28} \mathrm{H}_{21} \mathrm{NO}_{2}$ : C, 83.35; H, 5.25; N, 3.47. Found: C, 82.93; H, 5.40; N, 3.32.

\subsubsection{4,12-Dihydro-12,12-di(thien-2-yl)thieno[3',2':5,6][1,3] oxazepino[2,3-a]isoindol-} $\mathbf{6}(\mathbf{1 0 b} \boldsymbol{b})$-one (6d). This compound was prepared from $\mathbf{5 d}$ using the same procedure as for $\mathbf{6 a}$. Yield 100\%; mp 217 ${ }^{\circ} \mathrm{C}$; IR: $1703(\mathrm{C}=\mathrm{O}) \mathrm{cm}^{-1}$; ${ }^{1} \mathrm{H}$ NMR: $\delta 4.34\left(\mathrm{~d}, J=16 \mathrm{~Hz}, 1 \mathrm{H}, \mathrm{H}_{4}\right), 5.34(\mathrm{~d}$, $\left.J=16 \mathrm{~Hz}, 1 \mathrm{H}, \mathrm{H}_{4}\right), 6.14$ (s, 1H, $\left.\mathrm{H}_{10 \mathrm{~b}}\right), 6.80$ (dd, $J=4$ and $\left.2 \mathrm{~Hz}, 1 \mathrm{H}, \mathrm{H}_{\text {thiophene }}\right), 6.87-6.94$ (m, $\left.2 \mathrm{H}, \mathrm{H}_{3}+\mathrm{H}_{\text {thiophene }}\right), 6.95-7.07\left(\mathrm{~m}, 2 \mathrm{H}, \mathrm{H}_{\text {thiophene }}\right), 7.14\left(\mathrm{~d}, J=5 \mathrm{~Hz}, 1 \mathrm{H}, \mathrm{H}_{2}\right), 7.27$ (dd, $J=5$ and $\left.1 \mathrm{~Hz}, 1 \mathrm{H}, \mathrm{H}_{\text {thiophene }}\right), 7.40-7.59$ (m, 4H, 3 $\left.\mathrm{H}_{\text {arom }}+\mathrm{H}_{\text {thiophene }}\right), 7.78$ (d, J=6 Hz, $\left.1 \mathrm{H}, \mathrm{H}_{7}\right) ;{ }^{13} \mathrm{C} \mathrm{NMR}$ : $\delta 40.9\left(\mathrm{CH}_{2}\right), 82.1(\mathrm{C}), 84.5(\mathrm{CH}), 123.4(\mathrm{CH}), 123.4(\mathrm{CH}), 124.9(\mathrm{CH}), 125.5(\mathrm{CH}), 126.2$ $(\mathrm{CH}), 126.7(\mathrm{CH}), 126.9(\mathrm{CH}), 128.6(\mathrm{CH}), 129.0(\mathrm{CH}), 129.7(\mathrm{CH}), 130.0(\mathrm{CH}), 131.6(\mathrm{C})$, $132.1(\mathrm{CH}), 134.5$ (C), 142.2 (C), 144.4 (C), 146.0 (C), 150.2 (C), 166.7 (CO). Anal. Calcd for $\mathrm{C}_{22} \mathrm{H}_{15} \mathrm{NO}_{2} \mathrm{~S}_{3}: \mathrm{C}, 62.68 ; \mathrm{H}, 3.59 ; \mathrm{N}, 3.32$. Found: $\mathrm{C}, 62.88 ; \mathrm{H}, 3.68 ; \mathrm{N}, 3.46$.

\subsubsection{2,3-Dihydro-2-[2-(hydroxy-diphenylmethyl)thien-3-ylmethyl]-1H-isoindole-1,3-}

dione (7a). Compound 7a was synthesized using the same procedure as for the synthesis of 6a (starting from 5a or $\mathbf{6 a}$ ) but with $1 \mathrm{~mL}$ of TFA in place of PTSA and with a reaction time of 2 days: Yield 90\%; mp $150^{\circ} \mathrm{C}$; IR: $1718(\mathrm{C}=\mathrm{O}) \mathrm{cm}^{-1} ;{ }^{1} \mathrm{H}$ NMR: $\delta 4.62\left(\mathrm{~s}, 2 \mathrm{H}, \mathrm{CH}_{2}\right), 6.22$ (s, $1 \mathrm{H}, \mathrm{CH}), 7.02-7.26\left(\mathrm{~m}, 12 \mathrm{H}, \mathrm{H}_{\text {arom }}\right), 7.57-7.67$ (m, 2H, $\left.\mathrm{H}_{\text {phthalimide }}\right), 7.67-7.78(\mathrm{~m}, 2 \mathrm{H}$, $\left.\mathrm{H}_{\text {phthalimide }}\right) ;{ }^{13} \mathrm{C}$ NMR: $\delta 34.5\left(\mathrm{CH}_{2}\right), 49.2(\mathrm{CH}), 123.0(2 \mathrm{CH}), 123.8(\mathrm{CH}), 126.5(2 \mathrm{CH})$, 128.2 (4 CH), 128.7 (4 CH), $129.5(\mathrm{CH}), 131.8$ (2 C), $132.1(\mathrm{C}), 133.7$ (2 CH), 143.7 (2 C), 145.8 (C), 167.8 (2 CO). Anal. Calcd for $\mathrm{C}_{26} \mathrm{H}_{19} \mathrm{NO}_{2} \mathrm{~S}: \mathrm{C}, 76.26$; H, 4.68; N, 3.42. Found: C, $75.82 ; \mathrm{H}, 4.61 ; \mathrm{N}, 3.25$. 
(7b). Compound $\mathbf{7 b}$ was synthesized using the same procedure as for the synthesis of $\mathbf{7 a}$ (starting from 5b or $\mathbf{6 b}$ ). Yield 96\%; mp $176^{\circ} \mathrm{C}$; IR: $1710(\mathrm{C}=\mathrm{O}) \mathrm{cm}^{-1} ;{ }^{1} \mathrm{H}$ NMR: $\delta 4.77(\mathrm{~s}$, $\left.2 \mathrm{H}, \mathrm{CH}_{2}\right), 6.21(\mathrm{~s}, 1 \mathrm{H}, \mathrm{CH}), 6.76-6.90\left(\mathrm{~m}, 1 \mathrm{H}, \mathrm{H}_{\text {arom }}\right), 6.97-7.46\left(\mathrm{~m}, 13 \mathrm{H}, \mathrm{H}_{\text {arom }}\right), 7.56-7.69$ (m, 2H, $\mathrm{H}_{\text {phthalimide }}$ ), 7.69-7.81 (m, 2H, $\left.\mathrm{H}_{\text {phthalimide }}\right) ;{ }^{13} \mathrm{C} \mathrm{NMR}: \delta 39.2\left(\mathrm{CH}_{2}\right), 52.3(\mathrm{CH}), 123.2$ $(2 \mathrm{CH}), 126.2(2 \mathrm{CH}), 126.7(\mathrm{CH}), 127.8(\mathrm{CH}), 128.3(4 \mathrm{CH}), 129.6(4 \mathrm{CH}), 130.0(\mathrm{CH})$, $130.2(\mathrm{CH}), 131.9$ (2 C), 133.8 (2 CH), 134.4 (C), 141.9 (C), 143.6 (2 C), 168.1 (2 CO). Anal. Calcd for $\mathrm{C}_{28} \mathrm{H}_{21} \mathrm{NO}_{2}$ : C, 83.35; H, 5.25; N, 3.47. Found: C, 82.99; H, 5.05; N, 3.39.

\subsubsection{2,3-Dihydro-2-[2-(hydroxy-di(thien-2-yl)methyl)thien-3-ylmethyl]-1H-isoindole-}

1,3-dione (7d). Compound 7d was synthesized using the same procedure as for the synthesis of $7 \mathbf{a}$ (starting from $\mathbf{5 d}$ or $\mathbf{6 d}$ ) but the reaction time was 6 days: Yield $28 \%$; mp $159^{\circ} \mathrm{C}$; IR: $1713(\mathrm{C}=\mathrm{O}) \mathrm{cm}^{-1} ;{ }^{1} \mathrm{H}$ NMR: $\delta 4.76\left(\mathrm{~s}, 2 \mathrm{H}, \mathrm{CH}_{2}\right), 6.72(\mathrm{~s}, 1 \mathrm{H}, \mathrm{CH}), 6.84-6.95(\mathrm{~m}, 4 \mathrm{H}$,

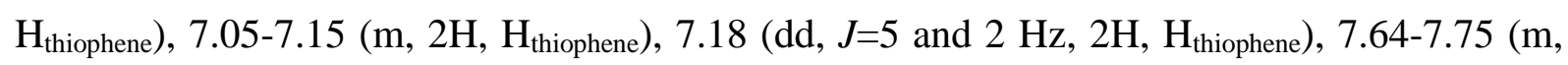
$\left.2 \mathrm{H}, \mathrm{H}_{\text {phthalimide }}\right)$, 7.76-7.86 (m, 2H, $\left.\mathrm{H}_{\text {phthalimide }}\right)$; ${ }^{13} \mathrm{C} \mathrm{NMR}: \delta 34.3\left(\mathrm{CH}_{2}\right), 40.0(\mathrm{CH}), 123.2(2$ $\mathrm{CH}), 124.0(\mathrm{CH}), 124.7(2 \mathrm{CH}), 126.0(2 \mathrm{CH}), 126.4(2 \mathrm{CH}), 129.4(\mathrm{CH}), 131.9(2 \mathrm{C}), 132.1$ (C), $133.8(2 \mathrm{CH}), 145.5(\mathrm{C}), 147.1(2 \mathrm{C}), 167.8$ (2 CO). Anal. Calcd for $\mathrm{C}_{22} \mathrm{H}_{15} \mathrm{NO}_{2} \mathrm{~S}_{3}$ : C, 62.68; H, 3.59; N, 3.32. Found: C, 62.78; H, 3.59; N, 3.24.

\subsubsection{3,3,4,4-Tetramethyl-1-[2-carbomethoxythien-3-ylmethyl]succinimide (8). A} mixture of tetramethylsuccinimide $(1.55 \mathrm{~g}, 10 \mathrm{mmol})$, crown ether 18-C-6 (0.01 g), potassium carbonate $(1.52 \mathrm{~g}, 11 \mathrm{mmol})$, potassium iodide $(0.166 \mathrm{~g}, 1 \mathrm{mmol})$, and dry toluene was stirred for $10 \mathrm{~min}$. A solution of methyl 3-bromomethylthiophene-2-carboxylate (2.82 g, $12 \mathrm{mmol}$ ) in toluene $(10 \mathrm{~mL})$ was added dropwise. The mixture was refluxed for $24 \mathrm{~h}$. After cooling, the mixture was filtrated on celite and was then concentrated under reduced pressure. The residue was recrystallized from ethanol to furnish pure 8 with a yield of $79 \%, \mathrm{mp} 154^{\circ} \mathrm{C}$; IR: 1696 $(\mathrm{C}=\mathrm{O}) \mathrm{cm}^{-1}$; ${ }^{1} \mathrm{H}$ NMR: $\delta 1.10\left(\mathrm{~s}, 12 \mathrm{H}, 4 \mathrm{CH}_{3}\right), 3.83\left(\mathrm{~s}, 3 \mathrm{H}, \mathrm{OCH}_{3}\right), 5.02\left(\mathrm{~s}, 2 \mathrm{H}, \mathrm{CH}_{2}\right), 6.77(\mathrm{~d}$, $\left.J=5 \mathrm{~Hz}, 1 \mathrm{H}, \mathrm{H}_{\text {thiophene }}\right), 7.36$ (d, J=5 Hz, $1 \mathrm{H}, \mathrm{H}_{\text {thiophene }}$ ). Anal. Calcd for $\mathrm{C}_{15} \mathrm{H}_{19} \mathrm{NO}_{4}: \mathrm{C}, 58.23$; H, 6.19; N, 4.53. Found: C, 58.04; H, 6.15; N, 4.35.

\subsubsection{3,3,4,4-Tetramethyl-1-[2-(diphenylhydroxymethyl)thien-3-ylmethyl]succinimide}

(9). This compound was obtained as an oil with a yield of $74 \%,{ }^{1} \mathrm{H}$ NMR: $\delta 1.10(\mathrm{~s}, 12 \mathrm{H}$, $\left.4 \mathrm{CH}_{3}\right), 4.30\left(\mathrm{~s}, 2 \mathrm{H}, \mathrm{CH}_{2}\right), 6.20(\mathrm{~s}, 1 \mathrm{H}, \mathrm{OH}), 6.82$ (d, J=5 Hz, 1H, $\left.\mathrm{H}_{\text {thiophene }}\right), 7.04$ (d, J=5 Hz, $\left.1 \mathrm{H}, \mathrm{H}_{\text {thiophene }}\right), 7.19-7.57\left(\mathrm{~m}, 10 \mathrm{H}, \mathrm{H}_{\text {phenyl }}\right) ;{ }^{13} \mathrm{C} \mathrm{NMR}: \delta 21.2\left(4 \mathrm{CH}_{3}\right), 36.5\left(\mathrm{CH}_{2}\right), 46.9(2 \mathrm{C})$, $124.9(\mathrm{CH}), 127.2(4 \mathrm{CH}), 127.3(2 \mathrm{CH}), 127.7(4 \mathrm{CH}), 128.6(\mathrm{CH}), 132.5(\mathrm{C}), 147.3(2 \mathrm{C})$, 
$149.5(\mathrm{C}), 183.2(2 \mathrm{CO})$.

\subsection{Reduction of imide 9, formation of $10 a+10 b$}

To a mixture of imide $9(1.73 \mathrm{~g}, 4 \mathrm{mmol})$ in dry methanol $(40 \mathrm{~mL})$ at $0^{\circ} \mathrm{C}$ was added sodium borohydride $(0.9 \mathrm{~g}, 24 \mathrm{mmol})$ by portions. To this mixture were added five drops of ethanolic hydrochloric acid solution (prepared from nine drops of concentrated hydrochloric acid in ethanol $(15 \mathrm{~mL})$ at regular intervals $(10 \mathrm{~min})$. The reaction was monitored by TLC. The excess of sodium borohydride was decomposed by careful addition of cold water $(15 \mathrm{~mL})$ and diluted hydrochloric acid. Sodium hydrogen carbonate was added and the solvent was evaporated. The residue was dissolved in a mixture of dichloromethane/water. The organic layer was dried on magnesium sulfate and was then concentrated under reduced pressure giving a mixture of $\mathbf{1 0 a} / \mathbf{1 0 b}(85 / 15)$ in a $95 \%$ yield.

\subsubsection{3,3,4,4-Tetramethyl-1-[2-(diphenylhydroxymethyl)thien-3-ylmethyl]succinamidal} (10a). ${ }^{1} \mathrm{H}$ NMR: $\delta 0.84\left(\mathrm{~s}, 3 \mathrm{H}, \mathrm{CH}_{3}\right), 0.88\left(\mathrm{~s}, 3 \mathrm{H}, \mathrm{CH}_{3}\right), 0.89\left(\mathrm{~s}, 3 \mathrm{H}, \mathrm{CH}_{3}\right), 1.03\left(\mathrm{~s}, 3 \mathrm{H}, \mathrm{CH}_{3}\right)$, $3.82(\mathrm{~m}, 1 \mathrm{H}, \mathrm{OH}), 4.04$ (d, J=15 Hz, 1H, $\left.\mathrm{CH}_{2}\right), 4.32$ (d, $\left.J=15 \mathrm{~Hz}, 1 \mathrm{H}, \mathrm{CH}_{2}\right), 4.53$ (d, J=5 Hz,

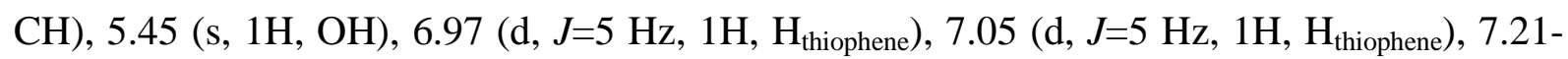
7.60 (m, 10H, $\left.\mathrm{H}_{\text {phenyl }}\right) ;{ }^{13} \mathrm{C}$ NMR: $\delta 17.4\left(\mathrm{CH}_{3}\right), 19.5\left(\mathrm{CH}_{3}\right), 22.4\left(\mathrm{CH}_{3}\right), 23.1\left(\mathrm{CH}_{3}\right), 38.2$ $\left(\mathrm{CH}_{2}\right), 42.4(\mathrm{C}), 46.0(\mathrm{C}), 78.9(\mathrm{CH}), 88.6(\mathrm{C}), 124.3(\mathrm{CH}), 127.2(2 \mathrm{CH}), 127.4(2 \mathrm{CH})$, $127.5(\mathrm{CH}), \quad 127.6(\mathrm{CH}), 127.8(2 \mathrm{CH}), 127.9(2 \mathrm{CH}), 130.0(\mathrm{CH}), \quad 134.8(\mathrm{C}), \quad 146.8$ (C), $147.0(\mathrm{C}), 147.8(\mathrm{C}), 180.0(\mathrm{CO})$.

\subsubsection{5-Methoxy-3,3,4,4-tetramethyl-1-[2-(diphenylhydroxymethyl)thien-3-ylmethyl]} succinamidal (10b). ${ }^{1} \mathrm{H}$ NMR: $\delta 0.85\left(\mathrm{~s}, 3 \mathrm{H}, \mathrm{CH}_{3}\right), 0.89$ (s, 3H, $\left.\mathrm{CH}_{3}\right), 0.94\left(\mathrm{~s}, 3 \mathrm{H}, \mathrm{CH}_{3}\right), 1.01$ (s, 3H, $\mathrm{CH}_{3}$ ), 3.44 (s, 3H, $\mathrm{OCH}_{3}$ ), 4.04 (d, J=15 Hz, 1H, $\mathrm{CH}_{2}$ ), 4.14 (d, J=15 Hz, 1H, $\mathrm{CH}_{2}$ ), 4.20 (s, 1H, OH), 6.98 (d, J=5 Hz, 1H, $\left.\mathrm{H}_{\text {thiophene }}\right), 7.03$ (d, J=5 Hz, 1H, $\left.\mathrm{H}_{\text {thiophene }}\right), 7.16-7.52$ (m, 10H, $\left.\mathrm{H}_{\text {phenyl }}\right)$.

\subsubsection{4,7,8,10-tetrahydro-7,7,8,8-tetramethyl-10,10-di-phenylpyrrolo[2,1-b]thieno[3,2-}

e] $[1,3]$ oxazepin-6(8aH)-one (11). This compound was prepared from the mixture of 10a and 10b using the same procedure as for the synthesis of $\mathbf{6 a , b}$. Yield $100 \%$; mp $157^{\circ} \mathrm{C}$; IR: 1665 $(\mathrm{C}=\mathrm{O}) \mathrm{cm}^{-1}$; ${ }^{1} \mathrm{H}$ NMR: $\delta 0.80\left(\mathrm{~s}, 3 \mathrm{H}, \mathrm{CH}_{3}\right), 0.98\left(\mathrm{~s}, 3 \mathrm{H}, \mathrm{CH}_{3}\right), 1.09$ (s, 3H, $\left.\mathrm{CH}_{3}\right), 1.31(\mathrm{~s}, 3 \mathrm{H}$, $\left.\mathrm{CH}_{3}\right), 3.85$ (d, J=15 Hz, 1H, $\mathrm{CH}_{2}$ ), 4.66 (s, $\left.1 \mathrm{H}, \mathrm{CH}\right), 5.14$ (d, J=15 Hz, 1H, $\left.\mathrm{CH}_{2}\right), 6.88-7.10$ 
(m, 2H, $\mathrm{H}_{\text {thiophene }}$ ), 7.12-7.46 (m, 10H, $\left.\mathrm{H}_{\text {phenyl }}\right) ;{ }^{13} \mathrm{C} \mathrm{NMR}: \delta 17.8\left(\mathrm{CH}_{3}\right), 18.4\left(\mathrm{CH}_{3}\right), 24.4$ $\left(\mathrm{CH}_{3}\right), 25.6\left(\mathrm{CH}_{3}\right), 41.0\left(\mathrm{CH}_{2}\right), 42.8(\mathrm{C}), 45.3(\mathrm{C}), 86.6(\mathrm{C}), 92.4(\mathrm{CH}), 124.5(\mathrm{CH}), 127.5(2$ $\mathrm{CH}), 127.7(2 \mathrm{CH}), 127.8(\mathrm{CH}), 128.4(\mathrm{CH}), 128.5(2 \mathrm{CH}), 128.9(\mathrm{CH}), 129.5(2 \mathrm{CH}), 136.3$ (C), 141.9 (C), 144.6 (C), 147.2 (C), 179.0 (CO). Anal. Calcd for $\mathrm{C}_{26} \mathrm{H}_{27} \mathrm{NO}_{2} \mathrm{~S}$ : C, 74.79; $\mathrm{H}$, 6.52; N, 3.35. Found: C, 74.35; H, 6.64; N, 3.31.

\section{References}

1. Cope, A. C.; Martin, M. M.; McKervey, M. A. Q. Rev. Chem. Soc. 1966, 20, 119-152.

2. March, J. Advanced Organic Chemistry: Reactions, Mechanisms, and Structure, Wiley: New York, 1992; pp 1062-1063 and references therein.

3. Prelog, V.; Kung, W. Helv. Chim. Acta 1956, 39, 1394-1406.

4. Hiemstra, H.; Speckamp, W. N. In Comprehensive Organic Synthesis, Trost, B. M., Fleming, I., Eds.; Pergamon: Oxford, 1991; Vol. 2, pp 1047-1082.

5. $\quad$ Pigeon, P.; Decroix, B. Synth. Commun. 1997, 27, 1423-1431.

6. Pigeon, P.; Decroix, B. Bull. Soc. Chim. Fr. 1997, 134, 153- 157.

7. Pigeon, P.; Othman, M.; Netchitailo, P.; Decroix, B. Tetrahedron 1998, 54, 14971506.

8. Corey, E. J.; Becker, K. B.; Varma, R. K. J. Am. Chem. Soc. 1972, 94, 8616-8618.

9. De Schryver, F. C.; Collart, P.; Vandendriessche, J.; Goedeweeck, R.; Swinnen, A.; Van Der Auweraer, M. Acc. Chem. Res. 1987, 20, 159-166.

10. Wiesler, W. T.; Nakanishi, K. J. Am. Chem. Soc. 1990, 112, 5574-5583. 